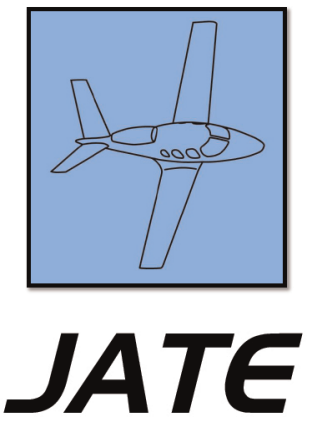

Journal of Aviation Technology and Engineering 1:2 (2012) 79-89

DOI: $10.5703 / 1288284314661$

\title{
A Comparison of the Effect of Variations to U.S. Airport Terminal Signage on the Successful Wayfinding of Chinese and American Cultural Groups
}

\author{
Steve Leib, Brian Dillman, Donald Petrin, and John P. Young \\ Purdue University
}

\begin{abstract}
Processing passengers quickly and efficiently is one of the top goals for airport administrators, and major international gateways have the added challenge of processing passengers representing a variety of cultures and nations. People with diverse cultural backgrounds may interpret signage layouts differently with respect to symbols and text. Consequently, dedicated research into signage perception may provide airport administrators with a more informed sense of how to convey appropriate movement and directional information within their terminals. There are many ways to answer the broad question of what signage encourages passengers to move most efficiently
\end{abstract}

\section{Introduction}

Arriving at a major airport from a long-haul international flight can be very stressful for passengers. Changes in time zone, fatigue, and discomfort of travel are all factors that can contribute to an unpleasant arrival experience. Because many people are in a physically and mentally weakened condition upon arrival, their goal is to be processed by Customs and

\section{About the Authors}

Steven Leib is currently a doctoral student in the Department of Aviation Technology at Purdue University. His present research is in Aviation Safety, specifically Safety Management Systems, and he is currently leading a joint international project in the US and China to assess Safety Management Systems in aviation organizations in China.

Brian Dillman is currently an Associate Professor in Aviation Technology at Purdue University. His current research activity has included developments in aircraft data collected from both engine and flight inputs, incident databases, preflight modification and assessments, situational judgment tests, and aeronautical decision making.

Donald A. Petrin is an Associate Professor in the Department of Aviation Technology at Purdue University where he has taught since 1979. His research interests have included crew resource management, aviation human factors, the cultural impact on aviation knowledge transfer; and VLJ training/ product support/airport emergency preparedness.

John Young is currently a professor in the Department of Aviation Technology at Purdue University. Professor Young's scholarly and research interests include aviation human factors and advanced cockpit training. Professor Young recently served as president of the University Aviation Association, representing over 100 college and university aviation programs. 
through an airport terminal. This study focused on the different ways in which Chinese and American airline passengers navigated through a terminal using either signs showing text only, symbols only, or both text and symbols. Quantitative in nature, the study used a unique computer simulation to generate paths having signage with English text, common symbols, or a combination of both. Participants clicked on arrows to follow the path to the specified goal for each different variation. Total completion time measurements and completion accuracy (in terms of correct versus incorrect clicks) were gathered. Analysis of the results suggested that there was a statistically significant difference related to cultural differences on a passenger's ability to wayfind based on the type of signage used. Additionally, within cultural groups there was a statistically significant difference as to which arrangements encouraged more efficient wayfinding and those which did not. Lastly, the results suggested a difference in which sign type produced the best wayfinding performance and which sign type people actually preferred.

Keywords: $\quad$ airport, sign, culture, Chinese, American, wayfinding

Immigration and proceed to other airport locations as quickly and smoothly as possible, with added urgency to passengers connecting to domestic flights.

Additionally, airport administrators have a vested interest in processing passengers in a quick and efficient manner. Given the daily influx of international travelers, airport administrators cannot afford to delay the process of moving passengers and baggage through their airports. There are many ways in which airports can bolster their passengers' ability to move through their terminals, and as travel has become more economical and available to more people worldwide, there are a variety of factors to consider when making decisions on the subject of airport wayfinding, the process by which one navigates an airport terminal using visual cues.

While the use of text, symbols, and/or directional arrows may seem intuitive and obvious to passengers from within American culture, passengers from different cultures may interpret those same instructional cues differently. Furthermore, signage arrangements may cause alternate interpretations from non-Americans. For major airports, especially those that have significant numbers of international commercial flights from U.S. and foreign airlines, displaying signs that best help the population of passengers move through the terminal is a goal that benefits everyone.

\section{Statement of the Problem}

As an increasing number of international passengers pass through U.S. airports, including the portion of Chinese passengers, airports have apparently done little to their facilities to reflect the changes in the cultural composition of their passengers. Aging facilities appear to have made few efforts to update signage, and airport administrators do not seem to consider the cultural background of their passengers when they do update signs. Because there is a fixed amount of walking room in terminals, passengers that cannot make effective use of signs can miss flights, cause unnecessary congestion, and waste their time as well as the time of the airport staff.

\section{Statement of Purpose}

The purpose of this study is to develop an understanding of how two different cultures, Chinese and American, utilize the content of terminal signage differently when navigating to the Customs \& Immigration area or other specified location of the airport upon landing at their United States gateway airport. This research narrows the many ways in which this issue can be addressed, and seeks to answer the following question: Is there a difference between the wayfinding abilities of Chinese and American airline passengers when they are presented with different signs using only English text, only symbols, or a combination of both?

This study compares the use of text and symbols combined with directional arrows to identify differences as to how Chinese and American passengers wayfind in U.S. airports, and provides a quantitative comparative analysis with qualitative support of how the two groups navigate differently based on the addition or removal of text and symbols on airport signs.

\section{Literature Review}

Fewings (2001) lays the groundwork for a problem that airports face every day: how to move passengers effectively through the terminal to get to various destinations. This can be described as "wayfinding," a term that defines the process of 
using environmental cues to successfully navigate to a particular destination. Fewings breaks wayfinding down into three different types, based on the reason for needing to reach a destination: recreation wayfinding, resolute wayfinding, and emergency wayfinding. Recreational wayfinding is considered the least urgent, where the navigator is wayfinding for their own enjoyment. For example, a simple "connect-the-dots" game or a stroll in a local park can be considered recreational wayfinding. Resolute wayfinding, however, has the goal of navigating as efficiently as possible. An example of this might be driving to a restaurant or walking to school, where the goal is to take the most efficient route. This is slightly different from emergency wayfinding. This type of wayfinding has the goal of getting to a particular destination as fast as possible. This could be a fire escape, or running from an attacker, and according to Fewings (2001), “...people will instinctively seek to use these [routes] under traumatic conditions" (p. 179). Wayfinding, according to Fewings, is the product of many visual cues, including signs (placement, color, content), terminal design (in the case of an airport), and maps of the area. Based on his research, he concludes that the different types of wayfinding command different types of information display. This naturally raises the issue of implementation of effective signage in airports, where wayfinding can be any of the three types depending on the circumstances, in such manner that a variety of situations is considered. This can be a major challenge for an airport authority.

Further challenges to airport wayfinding success come from the fact that airports are hosts to thousands of international passengers daily, and one of the fastest growing markets is passenger travel between the United States and China. The differences in these two cultures (as well as all of the other cultures represented in airports daily) present additional challenges to ensuring successful terminal navigation. In 2008, according to passenger data from the Office of Travel and Tourism Industries (OTTI), the United States received 493,000 Chinese visitors, which represents a $112 \%$ increase in the number of annual visitors from as recently as 2001. This is astonishing, especially given the fact that the Asian region as a whole had a $2 \%$ decrease in passengers over the same time interval. China also has the most impressive forecast for future growth in passenger exchange with the U.S. OTTI projections show that between 2008 and 2013, passengers from China will increase $61 \%$, which is the highest increase of any country in the world (the second highest being a $45 \%$ increase in passengers from Peru). This indicates a growing population of Chinese visitors in U.S. airports, which raises the question of cross-cultural wayfinding.

In addition, once in the United States, $50 \%$ of Chinese visitors report using the domestic aviation system, compared to only $25 \%$ of visitors from the Asian region as a whole (OTTI, 2008). In addition, 57.2\% of Chinese visitors went to the state of California. This indicates a strong presence of Chinese passengers in domestic and international airports as a fraction of international visitors, especially in California airports. This is especially significant because Los Angeles International Airport, San Francisco International Airport, and San Diego International Airport are ranked third, thirteenth, and twenty-seventh, respectively, on the list of busiest airports in the United States according to enplanements (FAA, 2008). It is perhaps because of this increasing presence of Chinese passengers that in 2007 the United States and China signed an agreement that will make group travel to the U.S. from China much easier. The agreement cites China as being the seventeenth largest international market for visitors to the U.S. in 2006, which was a record for exchange between the two countries. It also cites an $81 \%$ increase in passengers between 2006 and 2011. This natural growth in Chinese passengers, coupled with legislative encouragement from both governments, reinforces the need for airports to allow for multicultural wayfinding, especially with respect to Chinese culture given the sustained and predicted growth in the future.

One study on culture-based perception variation was conducted with Chinese and American university students to determine which part of warning signs induced the highest perceived hazard. Conducted by Lesch, Rau, Zhao, and Liu (2009), it evaluated students' responses in both countries based on three factors: sign color, text used, and symbol displayed. The researchers showed students different combinations of colors, words and symbols to convey the hazard, and students rated how high their perceived threat was. There were many results from the study, but one result relevant to understanding how Chinese people might perceive signage differently was that varying the symbols on signs that had the same color and word produced much greater fluctuations in perceived hazard for the Chinese students than the American students. "Therefore, in general, the language of the signal words did not have a large impact on perceived hazard-level." (Lesch, et al., 2009, p. 959). This result may be extremely relevant to airport signage because in this study the Chinese participants had a much deeper impact from the symbols used as opposed to the other factors (color and text) and could have strong implications as to how wayfinding can be improved in airports for Chinese passengers.

Differences in cognition appear to be correlated to cultural differences. Chen, Oyserman, Reber and Sorensen (2009) explore the theory of individualism versus collectivism in Chinese and Western cultures. They make three claims about the nature of cognition and how it can be affected. First, they argue that cognition is a product of context, and it subsequently can be shaped and molded based on environment. Furthermore, the conscious awareness of the person is not relevant in how that person's cognition is shaped; one does not need to be aware of a psychologically meaningful situation in order for it to affect one's cognition. Lastly, they 
describe how self-image has no bearing on cognitive development in a given context. This means that regardless of how a situation impacts one's self-image, there are distinct and separate effects on one's cognitive ability.

These claims have a distinct impact on how a difference in culture has a bearing on cognition, and furthermore how cognition manifests itself in terms of language and behavior. The researchers use this basis to show how two different cultures, Chinese and American, are collectivist and individualist cultures, respectively, but how situations can influence these mindsets to change cognition. They conducted eight experiments, each with cultures or sets of cultures, and found that although certain cultures are generally more individualistic or collectivistic, these cultural concepts are malleable, and can change based on context. For example, in one study Chinese students living in Canada were asked to describe their self-image in English and Chinese. The researchers found that when the students used Chinese to describe themselves, their answers were distinctly different from other students, but when they spoke English their answers related better to others'. The conclusion that the researchers drew was that the change in cognition (the switching of language) influenced a cultural change in that situation. These results could have useful implications in the airport planning and the development of airport signage, specifically the use of different languages on signs and how it might actually be a barrier on the group level, as it promotes cognitive difference in the situation of airport wayfinding.

The concept of human cognition in airport signs is especially important given that Boland, Chua, and Nisbett (2005) found distinct differences in the way people from Western and Chinese cultures perceived situations and how their focus was dissimilar. Their study was both qualitative and quantitative in determining this result. In some situations, they asked Chinese and Americans to describe an image and found that Americans tended to focus on the primary objects of the picture, and Chinese more thoroughly described the background scene, a more holistic approach to taking in a picture. Even more specifically, the quantitative side of the experiment measured the eye movements of the participants: "If differences in culture influence how participants actually view and encode the scenes, there will be differences in the pattern of saccades and fixations in the eye movements of the members of the two cultures" (Chua, et al, 2005, p. 12630). To distinguish the effect of culture on the observations of scenes, the researchers showed participants images a first time, and then changed the image backgrounds and presented the pictures to participants a second time. Measurements were then taken based on how much time the participant spent looking at the new background as opposed to the old foreground. Results indicated that "East Asians are less likely to correctly recognize old foregrounded objects when presented in new backgrounds...Thus, we have additional evidence for relatively holistic perception by East Asians: They appear to 'bind' object with background in perception" (Chua, et al, 2005 , p. 12632). This study can have additional implications on the layout and presentation of airport signs, especially since it shows how backgrounds are not just arbitrary to certain cultures, and can influence cognition in terms of understanding foregrounds. This may be an important concept in the development of airport terminal signs.

\section{Methodology}

There were two distinct samples in this study. The "American" group was comprised of people who:

1. Are U.S. citizens or U.S. permanent residents.

2. Speak English as their primary language and have learned English from birth.

3. Have not studied any ideographic or Asian languages.

4. Were born in the United States.

This population was considered Group 1, representing "American culture." The second population, representing "Chinese culture," was comprised of people who met the following qualifications:

1. Are citizens or permanent residents of the People's Republic of China or Taiwan.

2. Speak Mandarin Chinese or another Chinese dialect as their primary language.

3. Were born in the People's Republic of China or Taiwan.

Each group was a sample population representing the two cultures. In this study there were 20 participants in each sample population, representing different ages, genders, and geographical areas within the limits of the aforementioned definitions of the cultural groups.

\section{Data Collection}

All participants first confirmed that they were eligible for the study based on the specified qualifications. They then used a computer simulation that presented nine rounds of sign paths, each using one of three styles of signs:

1. A set of signs composed of symbols from the Professional Association for Design and directional arrows (AGIA, 2009). (Figure 1).

2. A set of signs composed of English text and directional arrows. (Figure 2).

3. A set of signs composed of both symbols and English text with directional arrows.

Participants were instructed that they had just arrived in the United States from an international flight and had to navigate to one of the five locations presented in all of the signs. Instructions were printed in both Chinese and English. Each round had ten signs, and participants advanced to the 


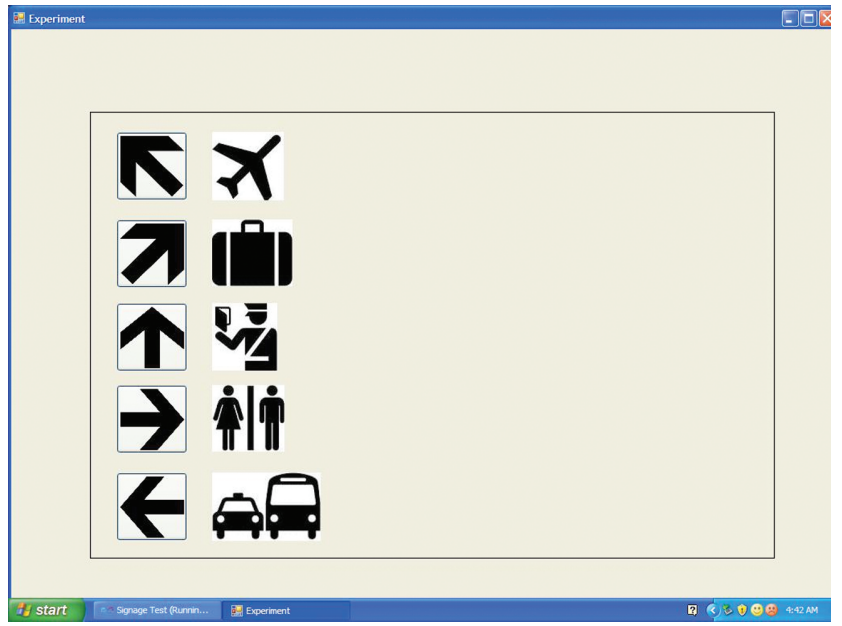

Figure 1. Screen shot from the computer simulation using the symbolsonly set of signs for a particular round.

next sign by clicking on the arrow corresponding to the correct location, which changed every round. Participants were timed from the start of the experiment until they successfully advanced through all ten of the signs of the given round. If they chose to, participants were allowed to pause for a few seconds between rounds.

The time each participant took to navigate each round was recorded and collected. If the participant clicked an arrow that indicated a destination other than the one specified for that round, the computer would produce an error message and require them to try again. In addition, the computer would make a note in the participant's file stating the round, sign, and which incorrect choice was made.

\section{Post-Simulation Survey}

Upon completing the ninth round, the participant was prompted to complete a short survey about what they experienced. The simulation then asked the participant five questions:

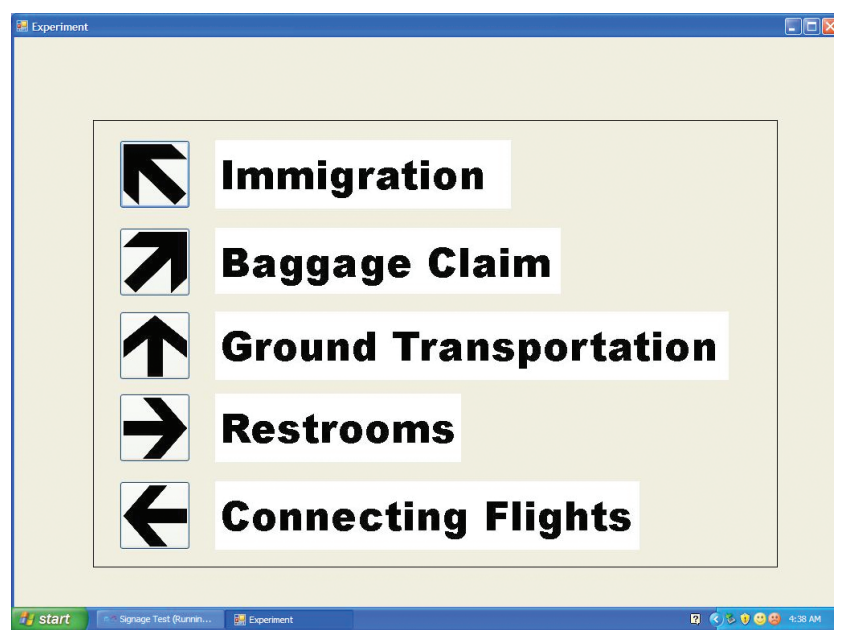

Figure 2. Screen shot from the computer simulation using the text-only set of signs for a particular round.
1. Which of the three sets of signs did they find to help them navigate the quickest?

2. An optional opportunity to explain their choice by typing English text.

3. How many times per year do they travel between a foreign country and the United States? (This question was multiple choice with options of 0-1, 2-3, or 4+).

4. Which symbols did the participant find confusing? (The program displayed all of the symbols chosen for the experiment and allowed the participant to check any number of them, including zero).

5. An opportunity to explain their choice[s] by typing English text.

All of this data was immediately transferred to that participant's file after each question. If they chose to type English text to explain their choices, their answers were recorded exactly as entered. The questions were displayed on the screen in English only but an accompanying document had both English and Chinese and could be used for reference.

\section{Data and Findings}

\section{Within-Group Analysis}

Because each participant was exposed to the same type of path three times (in terms of sign type) it was possible to isolate the round that had the shortest aggregate wayfinding time for each sign type. It is important to note that these were the rounds that had the shortest total wayfinding time for the group as a whole; the fastest rounds for each individual may have varied. For the Chinese participants, the aggregate wayfinding time was the shortest in round four for the text-only signs, round eight for the combination text-symbols signs, and round nine for the symbols-only signs, with aggregate totals of 378 seconds, 320 seconds, and 272 seconds, respectively. Figure 3 shows a visual representation of only those three data sets.

For the signs with symbols, the mean wayfinding time was 13.6 seconds, with a standard deviation of 7.85 . For the signs with the combination of text and symbols, the mean wayfinding time was 16 seconds with a standard deviation of 4.94, and for the signs with just text the mean wayfinding time was 18.9 with a standard deviation of 4.44. These were the results of the aggregate best performance rounds with $\mathrm{N}=20$ for each data set.

After identifying rounds four, eight, and nine as the rounds with the highest performance on the aggregate level, the next step was performing a statistical analysis of variance (ANOVA) to determine the likelihood that the variation of the data was not due to just random chance. ANOVA was acceptable for this set of data because the largest standard deviation of the three sets, 7.85, was smaller than twice the smallest standard deviation of the sets, 4.44. This, in turn, is based on the assumption of 


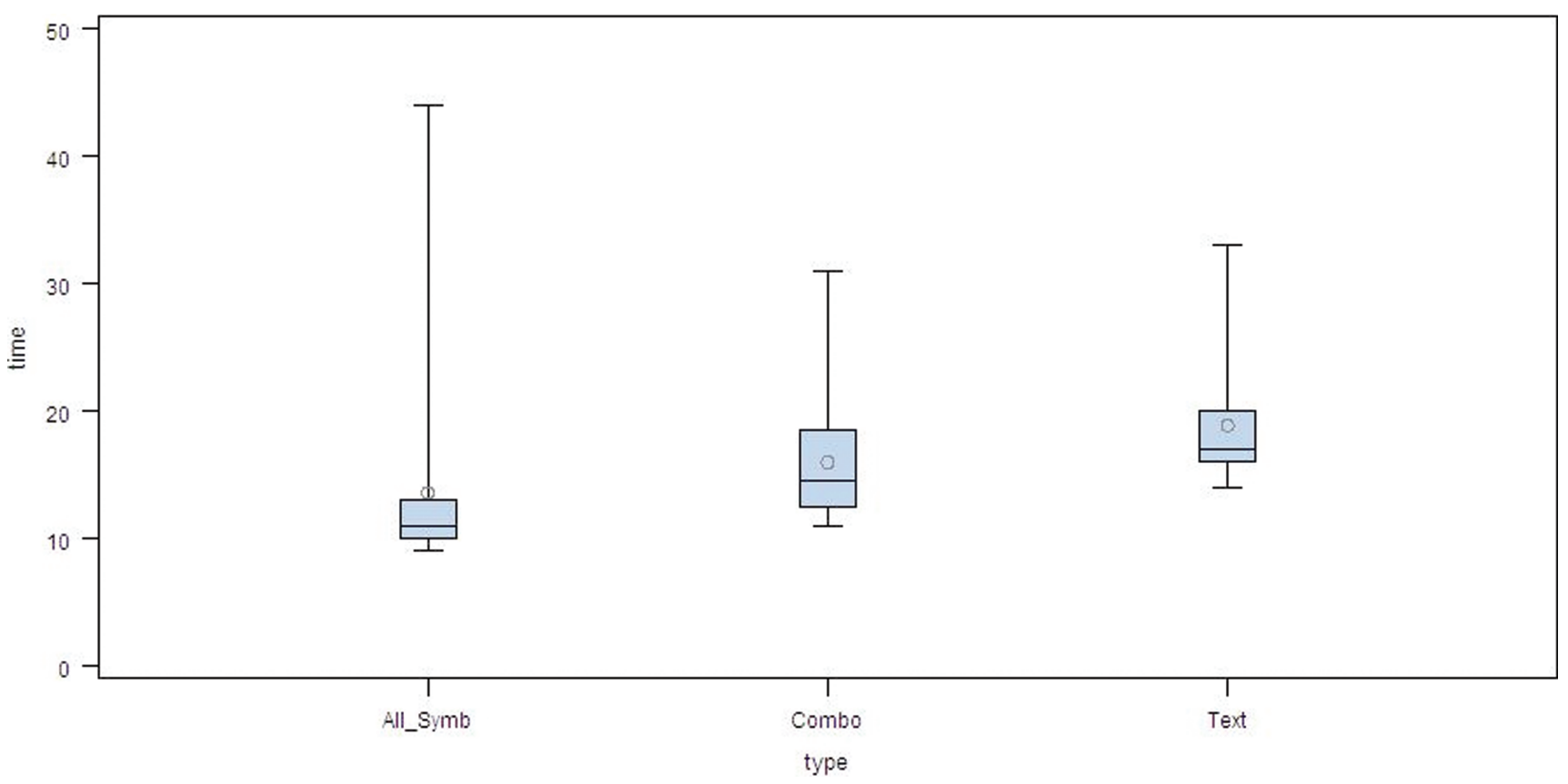

Figure 3 Aggregate data for the Chinese group for the best performance rounds of each sign type.

normality of the data sets, which is reasonably assumed for $\mathrm{N}>15$. $(\mathrm{N}=20$ in all three cases $)$.

First, it was necessary to hypothesize that the true means (as opposed to this data which shows sample means) of the each of the wayfinding times are the same for all the sign types. This null hypothesis, $H_{0}$, was used by the ANOVA to determine the likelihood that this particular set of data supported it.

The ANOVA produced a critical value $(\mathrm{F})$ of 4.00 , which, for two degrees of freedom, corresponded to a pvalue of 0.0237 . Furthermore, it produced a coefficient of determination $\left(R^{2}\right)$ of 0.1230 , which means that $12.30 \%$ of the variation of wayfinding times was directly due to the change in sign type.

Looking at the errors the participants made, there were a total of 21 errors. This indicates a point during the specified round where a participant clicked on a destination other than the assigned goal. Of the 21 errors, $52.38 \%$ of them (11) were made during the text-only sign rounds. Additionally, $19.04 \%$ of the errors (4) were made during the combination sign rounds, and $28.57 \%$ (6) were made during the symbols-only sign rounds.

Next was the same process as was done with the data from the Chinese participants; the rounds in which the American group performed the best were identified. Rounds seven at 265 seconds, eight at 271 seconds, and nine at 233 seconds, were the rounds in which the American group navigated the fastest for signs with text only, signs with the text-symbols combination, and signs with symbols only, respectively. Again, these are the rounds where the group performed best as a whole, and were not necessarily each individual participant's best round for that sign type. A visual display of the spread of wayfinding times for the best performance rounds is found in Figure 4. This set of data shows that for the best performance rounds, the American group had a mean wayfinding time of 11.65 seconds with a standard deviation of 2.54 for the symbols-only sign type, a mean wayfinding time of 13.55 seconds with a standard deviation of 2.19 for the combination text-symbols sign set, and a mean wayfinding time of 13.25 seconds with a standard deviation of 1.83 for the symbols-only sign type. These sets of data are assumed normal because $\mathrm{N}>15$ in all cases $(\mathrm{N}=20)$.

$H_{0}$ for the ANOVA of the American data was again the presumption that all of the true means are equal. The ANOVA was used to determine the likelihood that the sample means were a result of random chance as opposed to a rejection of $H_{0}$.

The ANOVA produced a critical value $(\mathrm{F})$ of 4.29 , which, for two degrees of freedom, corresponded to a $\mathrm{p}$ value of 0.0184 . The coefficient of determination $\left(R^{2}\right)$ from the ANOVA of the American data was 0.1308; meaning $13.08 \%$ of the variation of wayfinding times was directly due to the change in sign type.

Considering errors, the American group made a total of 13 mistakes in various rounds. Specifically, there were six errors $(46.15 \%)$ made during text-only rounds, although all of them were made in just the first round. Five errors, $(38.46 \%)$ were made in symbols-only rounds, and two errors $(15.38 \%)$ were made during the text-symbols combination rounds.

\section{Between-Groups Analysis}

On the aggregate level, the American group navigated faster than the Chinese group in every single round and 


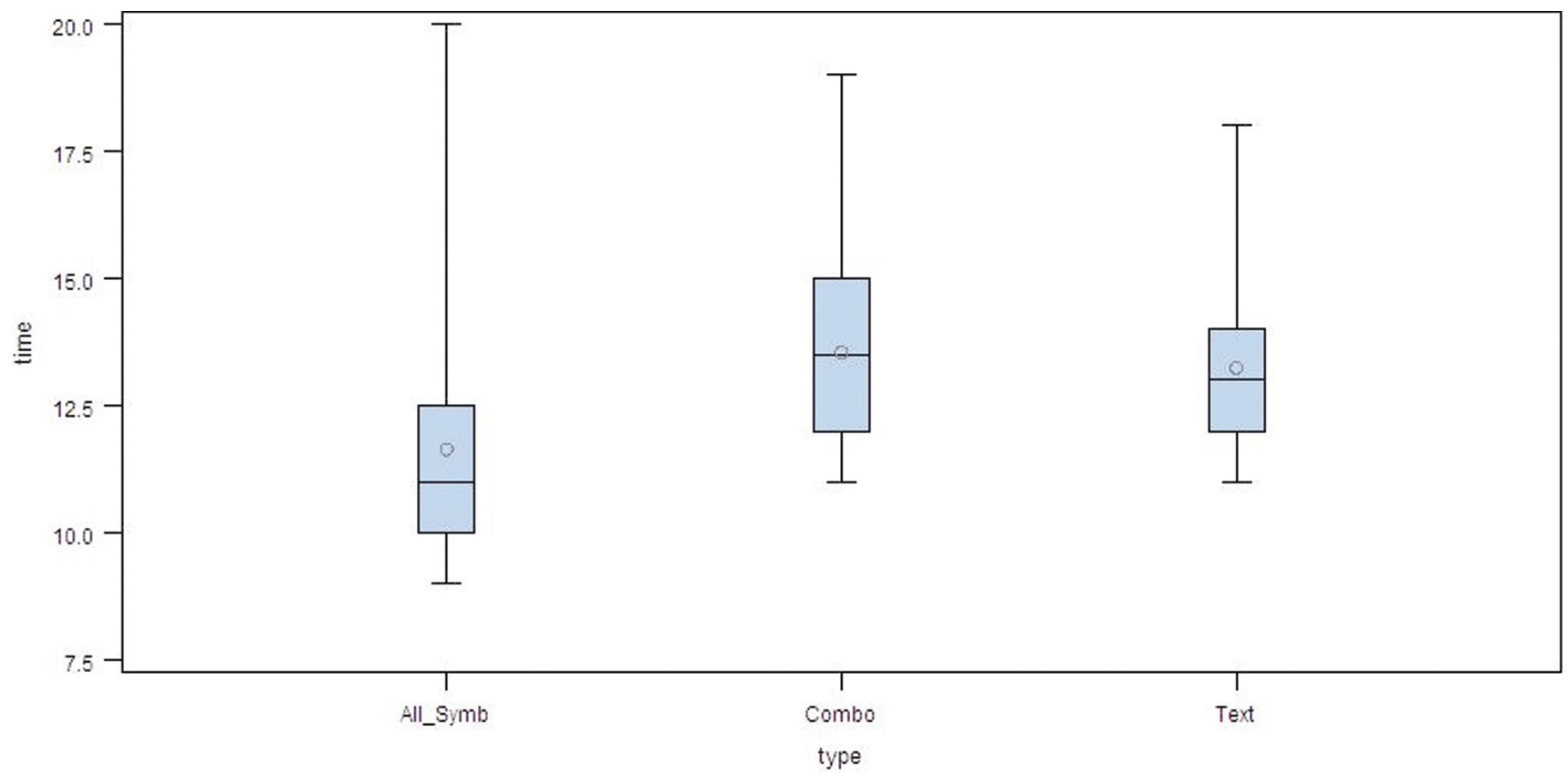

Figure 4. Aggregate data for the American group for the best performance rounds of each sign type.

therefore for every signage type as a whole. But to determine whether the differences in wayfinding times were statistically significant, a two-sample t significance test was used to compare the data. Below, Figures 5, 6, and 7 show a graphical comparison of the two groups' wayfinding times broken down by each sign type.

The results of the first two-sample $t$ significance test showed that in the case of the text-only sign type, the average means for the American and Chinese groups were 30.5 seconds and 46.18 seconds, respectively. The $p$ value for this data set was .0366.
The analysis of the data from the wayfinding times of the combination text-symbols sign set yielded means of 14.9 seconds and 18.55 seconds for the American and Chinese groups, respectively. The $\mathrm{p}$ value in this case was .0008 .

The last data set, the symbols-only sign set had means of 14.01 and 16.55 for the American and Chinese groups, respectively. Considering the closeness of the means and spread of the individual results, the associated $\mathrm{p}$ value for this set was .0496.

With respect to errors, the American group made fewer mistakes than the Chinese group. In fact, the 13 errors

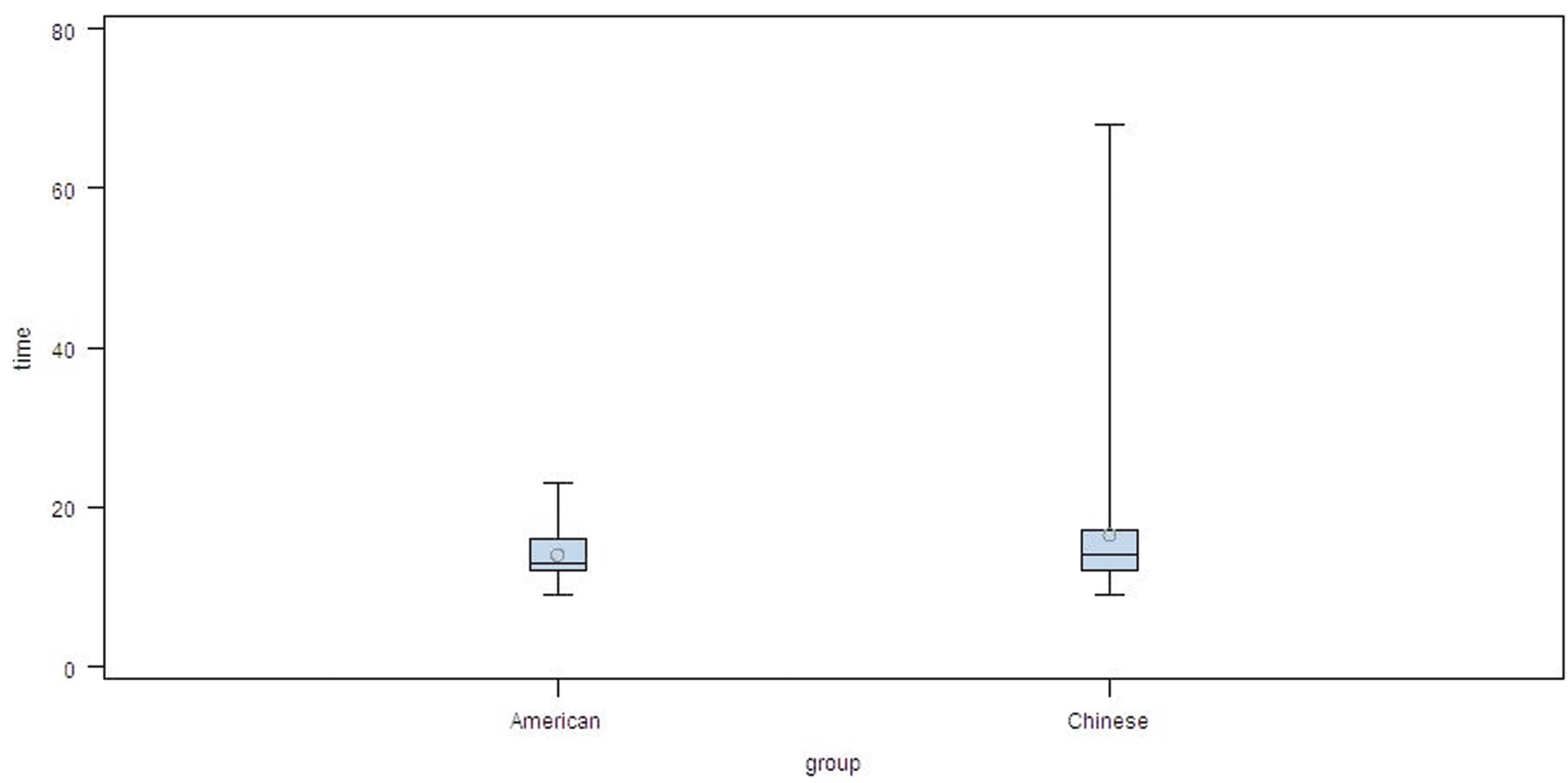

Figure 5. Aggregate wayfinding times of the Chinese and American groups for the symbols-only sign type. 


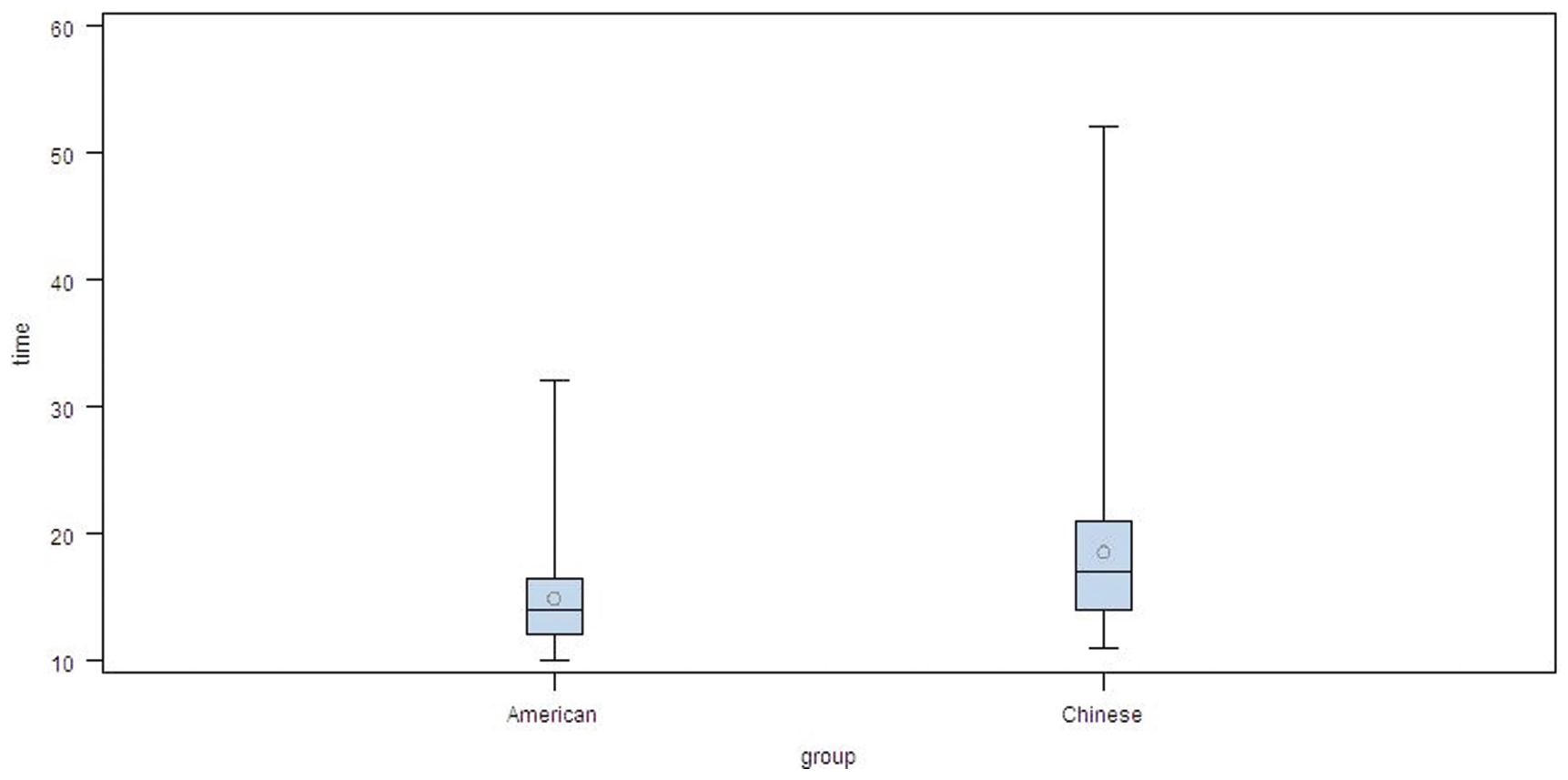

Figure 6. Aggregate wayfinding times of the Chinese and American groups for the combination text-symbols sign type.

made by the American group compared to the 21 errors made by the Chinese group mean that the Chinese group made $61.56 \%$ more errors than the American group.

After combining the total number from each group, it was possible to compare the aggregate number of errors of the American group to that of the Chinese group, both visually and statistically. Figure 8 , shown below, is a graphical comparison of the total number of errors made by each group and their spread of values.
Although there were only nine observations for each group, the standard deviations for the American and Chinese groups were 1.88 and 2.0, respectively. This, combined with the fact that the data is approximately normal despite $\mathrm{N}<15$, makes an ANOVA permissible.

The results of this test were a test statistic of .94 corresponding to a p-value of .3456 . The for this data set was .05575 , suggesting $5.58 \%$ of the variation in making errors was due to the cultural variation.

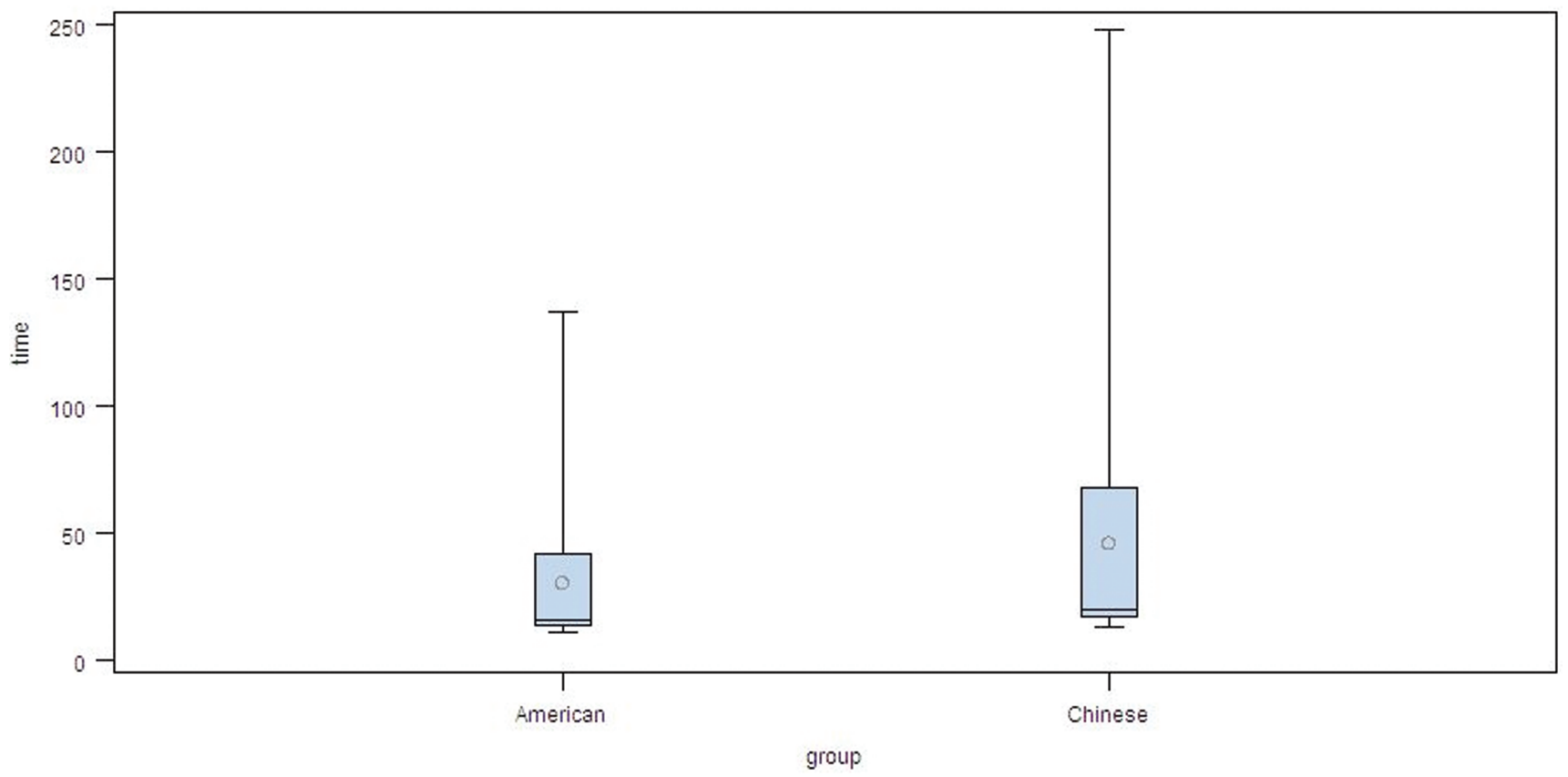

Figure 7. Aggregate wayfinding times of the Chinese and American groups for the text-only sign type. 


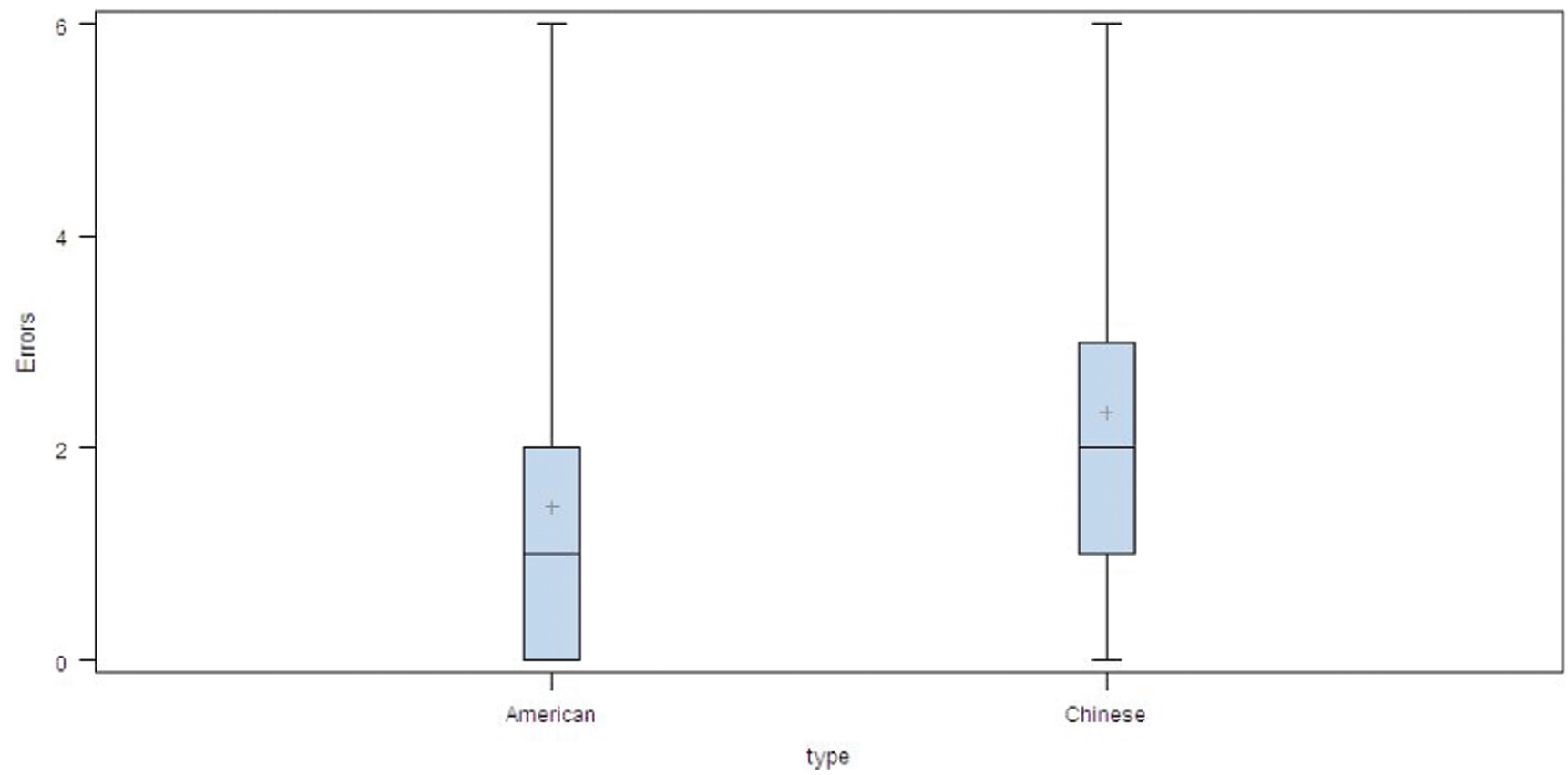

Figure 8. Aggregate error comparison between the American and Chinese groups.

\section{Qualitative Analysis}

A qualitative post-experiment survey provided the participants' opinions regarding which signs they found easiest to navigate as well as if they found symbols confusing, with an opportunity to explain why. They also reported the frequency of their international travel (to and from the United States).

Thirteen of the 20 Chinese participants felt that the textsymbols combination signs were the easiest to use to navigate. Within this group, many of the explanation responses indicated that they preferred the multiple information media so that one can help clarify the other if it was not clear on its own. One participant who chose the combination signs wrote, "Because the signs with pictures is [sic] easier for people to recognize and text can let me make sure I am not misunderstanding what the signs mean." Most of the other participants that chose this option did so not because they had trouble understanding the text or symbols, but because they enjoyed the further clarification.

Of the five that preferred the symbols-only sign type, the general response was that they understood the signs, and therefore regarded the text as unnecessary information. In this group, there was only one response second question about which symbols they found confusing - this group appeared to have a solid understanding of what each symbol meant.

The two people that preferred the English text did so because they did not feel the symbols were intuitive and added confusion to the wayfinding process. Both agreed that the symbols were hard to associate with their idea of the goal, and one even commented that many people would not know the symbols, especially if it was their first time traveling.

Regarding recognition of the symbols themselves, the two aforementioned participants seemed to be on the extreme end of recognizing and understanding the symbols. Generally, participants indicated that they found some symbols confusing and others not confusing, and there were two obvious trends indicated by the data. First, the immigration symbol was the most confused, with eight of the 20 participants in agreement. Their feelings were that it did not really embody the concept of immigration. Two participants actually felt that the symbol indicated police, or a police station. Many of the other participants simply could not discern its meaning without the aid of the text. One of them wrote, "if it is the first time to meet, you [sic] have no idea what they mean".

The other trend was a fair amount of confusion toward the "connecting flights" symbol. Participants that found this confusing, of which there were six, felt that there was a problem with the absence of another plane. Many of them wrote that there ought to be two planes to indicate a transfer, and some felt that that particular symbol would be better to indicate departures or arrivals.

The American group had results very similar to that of the Chinese group, but was stronger in the sense that they had more convincing numbers to emphasize their choices and opinions.

An overwhelming majority, 14 of the 20 participants (70\%), chose the combination text-symbols sign type as their preferred sign type. This group almost unanimously agreed that it was better to have more information on the sign so that they could look at either the text or the symbols, and if necessary have one help explain the other. 
One American participant wrote, "You can read the words or recognize the picture. There are two ways to recognize the right answer." Many other participants agreed and wrote very similar responses.

Five participants chose the text-only sign type, and generally agreed that they preferred not having the added distraction of trying to figure out the meaning of a symbol when the word was all that was necessary. One even felt that in order to understand the symbol they had to convert the symbol back into words anyway. Participants also indicated that they felt many of the symbols were confusing, and overall were more distracting rather than helpful in assisting them with wayfinding.

In American group, only one participant indicated a preference of the symbols-only sign type for wayfinding. One participant simply wrote, "Reading takes longer".

Regarding the symbols themselves, the American group was also very similar to the Chinese group in their opinions about which signs were confusing. More than half (11 out of 20) of the participants agreed that the symbol for "immigration" was confusing. They cited the same reasons as the Chinese group, explaining that the man in the symbol was ambiguous, the symbol could indicate a lot of things other than "immigration," and that nothing about that particular symbol made them think about immigration.

Additionally, the second most confusing symbol according to the American group was the symbol for "connecting flights," as was the case with the Chinese group. Again, the reasoning is very similar. Participants commented that there was just one airplane in the image, or that it could mean a variety of different things. Five participants of the 20 felt this way.

Comparing the two groups as a whole, both the Chinese and American groups had very similar responses to the survey questions. Regarding frequency of travel, all participants except for a handful in the Chinese group indicated that they travel internationally to or from the United States once or less per year.

One response, or lack thereof, that stands out strongly was the complete absence of the "restrooms" symbol. Not one participant in either group found that symbol confusing. While every other symbol was considered confusing by at least one participant of the 40 total, it appeared that everyone understood the indication for the restrooms.

\section{Conclusions}

For the purposes of determining statistical significance, an alpha level of $\alpha=.05$ was used as the significance threshold, a commonly accepted value in the field of statistics to describe data as statistically significant (Craig, et al., 2009).

It is possible to draw a number of reasonable conclusions from this study. Considering first the data from within the groups, the Chinese group navigated each signage path with an analysis of variance p-value of .0237 - different enough wayfinding times to show statistical significance. This means that there was indeed a clear difference in wayfinding speeds for the Chinese group among the three different sign types, and the data from this sample likely reflects the greater population as a whole.

This set of results is somewhat similar to the preferences expressed in the post-experiment survey. The Chinese overwhelmingly preferred the combination text-symbols sign type, followed by the symbols-only sign type, and lastly the textonly sign type. While it is certainly no surprise that the textonly sign type was both the slowest to navigate and the least preferred, it is interesting to note that while the Chinese performed best for the symbols-only sign type, they preferred the combination text-symbols sign type to navigate. This may indicate a higher value in wayfinding accuracy rather than time as a more important goal for passengers in airport terminals, as they also made more errors in the symbols-only sign type.

The results from within the American group are similar. The p-value for the ANOVA of the wayfinding times was .0184 , far less than the commonly accepted .05 significance level. This means that the difference in wayfinding speeds reflected in this study are also very likely to mirror those of the American population as a whole.

The survey responses from the American group were a bit surprising. Like the Chinese, Americans preferred the combination text-symbols sign type overwhelmingly, but unlike the Chinese, the Americans performed (with statistical significance) the absolute worst for this sign type. The fact that Americans prefer the sign type that leads to their slowest wayfinding, combined with the preference of the Chinese participants, corroborates the idea that airport terminal passengers value the accuracy of their wayfinding over the time. This is likely because despite it being their worst performance in terms of time, it turned out to be their best performance in terms of accuracy. Only 2 errors were made on the aggregate level for the combination text-symbols sign type, as opposed to the 5 errors made on the aggregate level for the symbols-only sign type (with which they navigated the fastest).

With a p-value of .3456, the difference in errors measured by each group was not statistically significant. Although this potential is more likely than not $65.5 \%$ more likely) there was not strong enough likelihood to reject $H_{0}$, which was that the true means of error rates between the two groups were equal to each other. It is therefore inconclusive as to, on the aggregate level, whether Americans wayfind with more accuracy than Chinese.

An analysis of how the groups compared to each other for each sign type showed that, in all three sign types presented in this study, the American group navigated faster than the Chinese group. For the text-only sign type, Americans navigated faster than Chinese with a $p$-value of .0366. For the combination text-symbols sign type, Americans navigated faster, with a p-value of .0008 . 
Lastly, for the symbols-only sign type, the American navigated faster with a p-value of .0496. All of these pvalues are less than the $\alpha=.05$ level, and are therefore statistically significant. The most interesting of these values is perhaps the p-value for the combination text-symbols sign type comparison. This value, .0008 is so small that it suggests strongly that as a whole, for signs that have both English text and symbols, Americans wayfind faster than Chinese. Interestingly enough, this is even more significant than the p-value for the comparison of the English text-only wayfinding times. This implies that the addition of symbols to an airport terminal sign makes the Chinese group wayfind faster, and the American group navigate slower (both with statistical significance as described above), but at the same time solidifies the extreme likelihood that in that situation, the Americans will still navigate faster, in an American airport. However, because all three of the pvalues for the cross-cultural comparisons are less than $\alpha=.05$, it is a reasonable conclusion that no matter what the usage of English text and/or symbols, Americans will be able to navigate faster in an American airport terminal.

\section{Recommendations}

Part of the conclusion of this study is that there is a distinct difference between what sign type people prefer and with what sign type people perform the best. This puts airport administrators who make decision of what type of signs to put in their terminals in a unique position. They can either value what will move people through their terminals the quickest or value what people prefer, regardless of their performance. It is therefore recommended that airport administrators determine their hierarchy of values for their airports. Ranking values such as wayfinding time, passenger preference, and wayfinding accuracy will help guide an airport administrator as to which sign type will be the best fit for their airport.

In addition, airports should also begin to consider the future and current cross-section of passengers their airports encounter daily. This study focused on the cultural influence of Chinese and Americans, but comparing performance and preferences of other cultural groups may yield different results. For example, this study specifically may be more applicable to airports on the west coast of the United States, but airports in the American south and east ought to consider their own cultural cross-section based on their flight frequency to Latin America and Europe. From this perspective, perhaps a national sign type standard is not the direction toward which airport administrators should aim. Instead, it may be more beneficial to airports to have sign types that meet their own passengers' needs best, which may differ geographically. This, however, only applies to sign layouts, and is not a recommendation for choosing symbols to indicate various destinations on the signs themselves. The post-experiment survey in this study showed that both cultural groups felt very similarly about which symbols were confusing and which were clear, and having varying symbols to explain the same locations at different airports is not a recommendation encouraged by these results. Overall, the results can be summarized in four major points:

- There is a statistically significant difference among wayfinding speeds for each of the three sign types for both the American and Chinese groups.

- The American group navigated significantly faster than the Chinese group, on the aggregate level, for every sign type.

- There was a clear difference for both groups between what sign type was best for wayfinding and what sign type was preferred by most of the group.

The results indicated that there is indeed an influence of culture on how people wayfind, and there is certainly further study that can be done to explore the extent of this influence and how other factors play a role in successful wayfinding.

\section{References}

AIGA (2009). Retrieved from the Professional Association for Design website: http://www.aiga.org.

Chen, S., et al. (2009). Connecting and separating mind-sets: Culture as situated cognition. Journal of Personality and Social Psychology 97(2), 217-235, doi: 10.1037/a0015850.

Chua, H., et al. (2005). Cultural variation in eye movements during scene perception. Proceedings of the National Academy of Sciences of the United States of America 102(35), 12629-12633, doi: 10.1073/pnas. 0506162102.

Craig, B., et al. (2009). Introduction to the practice of statistics (6th ed.). New York, NY: W.H. Freeman and Company.

Fewings, R. (2001). Wayfinding and airport terminal design. The Journal of Navigation, 54(2), 177-184.

Lesch, M., Rau, P., Zhao, Z., \& Liu, C. (2009). A cross-cultural comparison of perceived hazard in response to warning components and configurations: U.S. vs. China. Applied Ergonomics, 40, 953-961, doi: 10.1016/j.apergo.2009.02.004.

Office of Travel and Tourism Industries (2007). Fact Sheet U.S.-China Group Leisure Travel memorandum of Understanding (MOU). Retrieved from http://tinet.ita.doc.gov/pdf/MOU.pdf

Office of Travel and Tourism Industries. Latest Statistics/Outreach [Data files]. Retrieved from http://tinet.ita.doc.gov/outreachpages/index.html

Office of Travel and Tourism Industries. Research Programs [Data files]. Retrieved from http://tinet.ita.doc.gov/research/monthly/index.html

Office of Travel and Tourism Industries. (2009). 2008 Market Profile: Asia. Retrieved from http://www.tinet.ita.doc.gov/outreachpages/ download_data_table/2008_Asia_Market_Profile.pdf

Office of Travel and Tourism Industries. (2009). 2008 Market Profile: China. Retrieved from http://www.tinet.ita.doc.gov/outreachpages/ download_data_table/2008_Asia_Market_Profile.pdf

Office of Travel and Tourism Industries. (2009). International Travel Forecast. Retrieved from http://tinet.ita.doc.gov/view/f-2000-99-001/ forecast/Forecast $\% 20$ Summary.pdf 